\title{
Differential Expression and PAH Degradation: What Burkholderia vietnamiensis G4 Can Tell Us?
}

\author{
Guilherme Pinto Cauduro (D), ${ }^{1}$ Ana Lusia Leal, ${ }^{2}$ Tiago Falcón Lopes, ${ }^{3}$ Marcela Marmitt, ${ }^{1}$ \\ and Victor Hugo Valiati $\mathbb{D}^{1}$ \\ ${ }^{1}$ Universidade do Vale do Rio dos Sinos, Biology Graduate Program, São Leopoldo, RS, Brazil \\ ${ }^{2}$ Companhia Riograndense de Saneamento, Biology Laboratory, Triunfo, RS, Brazil \\ ${ }^{3}$ Centro de Terapia Gênica, Centro de Pesquisa Experimental, Hospital de Clínicas, Porto Alegre, RS, Brazil
}

Correspondence should be addressed to Guilherme Pinto Cauduro; gcauduro@gmail.com and Victor Hugo Valiati; valiati@ unisinos.br

Received 1 April 2020; Revised 4 July 2020; Accepted 31 July 2020; Published 27 August 2020

Academic Editor: Todd R. Callaway

Copyright (C) 2020 Guilherme Pinto Cauduro et al. This is an open access article distributed under the Creative Commons Attribution License, which permits unrestricted use, distribution, and reproduction in any medium, provided the original work is properly cited.

\begin{abstract}
Petroleum is the major energy matrix in the world whose refining generates chemical byproducts that may damage the environment. Among such waste, polycyclic aromatic hydrocarbons (PAH) are considered persistent pollutants. Sixteen of these are considered priority for remediation, and among them is benzo(a)pyrene. Amid remediation techniques, bioremediation stands out. The genus Burkholderia is amongst the microorganisms known for being capable of degrading persistent compounds; its strains are used as models to study such ability. High-throughput sequencing allows researchers to reach a wider knowledge about biodegradation by bacteria. Using transcripts and mRNA analysis, the genomic regions involved in this aptitude can be detected. To unravel these processes, we used the model B. vietnamiensis strain G4 in two experimental groups: one was exposed to benzo(a) pyrene and the other one (control) was not. Six transcriptomes were generated from each group aiming to compare gene expression and infer which genes are involved in degradation pathways. One hundred fifty-six genes were differentially expressed in the benzo(a)pyrene exposed group, from which 33\% are involved in catalytic activity. Among these, the most significant genomic regions were phenylacetic acid degradation protein paaN, involved in the degradation of organic compounds to obtain energy; oxidoreductase FAD-binding subunit, related to the regulation of electrons within groups of dioxygenase enzymes with potential to cleave benzene rings; and dehydrogenase, described as accountable for phenol degradation. These data provide the basis for understanding the bioremediation of benzo(a)pyrene and the possible applications of this strain in polluted environments.
\end{abstract}

\section{Introduction}

Polycyclic aromatic hydrocarbons (PAHs) have been increasingly released into the environment by incomplete combustion of organic materials. This group of contaminants has harmful biological effects such as carcinogenicity, mutagenicity, and genotoxicity $[1,2]$ and it stands out as some of the most persistent pollutants in nature. They are classified as Persistent Organic Pollutants (POPs) of which 16 are recognized as priority for remediation by the US Environmental Protection Agency (USEPA) for having high toxicity levels to human health [1], including the benzo(a) pyrene used in this study, characterized by their stability and difficult degradation due to their chemical structure (aromatic rings) and their hydrophobicity [3-5]. Despite these properties, as they have genes that are expressed differently in the presence of these compounds $[6,7]$, a variety of bacteria can use them as a source of carbon and energy through their own metabolic pathways, playing a role in recycling the carbon of aromatic rings [8], degrading such pollutants [1].

Studies have shown that the enzymes, proteins, and metabolic pathways responsible for the biodegradation of PAHs can be produced in larger quantities, thus increasing 
the energy gain of these bacteria and their survival rate, making them adaptable to these environments [9-12]. De Gannes [13], in his study on genetic adaptations, presents three ways in which bacteria can survive in an environment with PAHs, such as carbon assimilation mediating enzymes, regulatory pathways for the expression of these enzymes, and modifications in cell structures for the absorption of compounds. Differential expression analysis and bioremediation experiments using these microorganisms allow us to detail this adaptation processes [14-17].

The Burkholderia cepacia complex (BCC) has been used as a model for the study of biodegradation [18]. This complex has at least nine species: $B$. cepacia, B. multivorans, B. cenocepacia, B. vietnamiensis, B. stabilis, B. ambifaria, B. dolosa, B. anthina, and B. pyrrocinia, which are genetically distinct and phenotypically similar [19]. They present great phenotypic plasticity and ecological versatility due to the size of their genome (4-9 Mb) [19]. Burkholderia vietnamiensis G4 [19] has been associated with organic pollutants since 1986, when its ability to metabolize trichloroethylene was recognized [20]. It has biotechnological potential and is used as a model of biodegradation due to its ability to degrade benzene, o-cresol, p-cresol, phenol, toluene, chloroform, benzo(a)pyrene, and naphthalene [21-24].

The $B$. vietnamiensis G4 capacity to survive harmful compounds in contaminated areas allowed its use in bioremediation experiments, combined with high-performance sequencing techniques and transcription analysis, which enabled the identification of genes differently expressed in its genome [23, 25-27] and its functions in the biodegradation process [28-31]. The use of new high-performance sequencing tools and transcriptome analysis is important to help us understand microorganism responses under different stress conditions [32]. In this context, the present study performed transcriptomic analyses in populations of Burkholderia vietnamiensis G4 exposed to benzo(a)pyrene with the aim to reveal the genes related to the degradation of this compound.

\section{Materials and Methods}

2.1. Bacterial Growth and RNA Extraction. The model selected for the differential expression tests was Burkholderia vietnamiensis strain G4 (Gram-negative, aerobic). This bacterium was chosen because it has already been described as presenting biodegradation capacity of several organic compounds. Its genome has been fully sequenced, which facilitates and enables a more detailed exploration of the data obtained with the differential gene expression experiment.

As a model for PAH, benzo(a)pyrene was chosen because it is one of the priority pollutants for remediation; it has five benzene rings making it exceedingly difficult to degrade, and it has high carcinogenic potential.

Initial bacterial growth was performed in liquid LB culture medium for 24 hours. Subsequently, the number of bacteria of $10^{8}$ cells $/ \mathrm{mL}$ was standardized, using a spectrophotometer and absorbance reading at $625 \mathrm{~nm}$. From this culture, the experimental stage of exposure of the bacteria to benzo(a)pyrene was initiated. Six Erlenmeyer culture flasks with $150 \mathrm{~mL}$ of Minimum Mineral Medium enriched with $10 \mathrm{mg} / \mathrm{L}$ of glucose were used, on which $1 \mathrm{~mL}$ of bacterial culture was added. In three of these, $1 \mathrm{mg} / \mathrm{L}$ of benzo(a) pyrene was added-characterizing the experimental group exposed to PAH (Bap1, Bap2, and Bap3). In the other three cultures, there was no addition of benzo(a)pyrene, and these were considered an unexposed control group (Neg1, Neg2, and Neg3) for expression comparisons. Both cultures, experimental and control, were incubated for 45 minutes at $30 \mathrm{o} C$ and shaken at $140 \mathrm{rpm}$. The time of exposition was set to allow the bacteria to enter the growth phase, but not reach the duplication phase, thus avoiding production of RNAs involved in cell duplication.

After 45 minutes, all cellular processes were interrupted with the ProtectCell ${ }^{\circledR}$ kit (QIAGEN, Germany), increasing the stability of the extracted materials. The extraction of total RNA was performed with the RNeasy Mini Kit ${ }^{\circledR}$ (QIAGEN, Germany), following the manufacturer's recommendations. For each culture, two pools of total RNA were extracted and enriched with the Ribo-Zero rRNA Removal Kit-Bacteria ${ }^{\circledR}$ (Illumina ${ }^{\circledR}$ ), producing twelve mRNA libraries, six from the experimental group and six from the control group.

2.2. RNA Sequence (RNA-Seq). The sequencing of the twelve RNA libraries was performed on the single-end Illumina ${ }^{\circledR}$ (MiSeq) platform. The libraries were built using specific tags, following the manufacturer's instructions (TruSeq TMRNA $^{\circledR}$ (Illumina $\left.{ }^{\circledR}\right)$ ).

2.3. Library Analysis. For the library analysis, samples originated from the same Erlenmeyer flask (two sequences) formed a single sample unit, with three experimental samples exposed to benzo(a)pyrene and three nonexposed control samples.

Sequences were filtered considering the Phred quality $\geq 30$, which represents a $99 \%$ hit in the recognition of the bases. The Phred quality system is calculated by the probability of a base having been erroneously sequenced, given by the formula $q=-10 \times \log (p)$, where $p$ represents such probability. If an error is expected every one thousand sequenced bases, the Phred 30 value represents the expected error estimated at sequencing [33]. The quality verification of the filtered reads was performed with FastQC software version $0.11 .3[34,35]$. The reads were mapped by alignment with TopHat program version 2.0.9 (http://tophat.cbcb.umd. edu), which uses the files generated in the sequencing, aligning the reads in the indexer assembled with the genome (GenBank assembly accession: GCA_000016205.1) of the bacteria and its annotation file through Bowtie software version 1.1.1 (http://bowtie-bio.sourceforge.net/index. shtml).

2.4. Differential Expression Analysis. The quantification of the genes involved in the degradation capacity of benzo(a) pyrene, after alignment with TopHat, was performed with the software Cufflinks v. 2.1.1 (http://cufflinks.cbcb.umd. edu) [34]. The Cuffmerge extension was used to integrate 
the reads to TopHat mapping. Then, through the Cuffdiff tool, the levels of gene expression were verified and the statistical significance for the experimental and control groups was tested. Using the R (v. 3.3.2) package, CummeRbund version 0.1.3 (http://compbio.mit.edu/cummeRbund/), the analysis regarding the levels of expression was accessed [36]. To measure the significance of the differential expression, the corrected $p$ values ( $q$-value) were used, considering the False Discovery Rate $(\mathrm{FDR})<0.05$. All plots and relationship evaluations of the genes significantly differentially expressed were generated with the gplots package.

Clusters analysis was performed using the pvclust package version 2.0.0 [37], based on the correlation distance associated with a complete connection method with 1000 replications of the bootstrap type. Correlation methods are highly recommended for gene expression data [38]. To verify the significance measurements, the corrected $p$ values and the bootstrap were used as significance measurements, considering as significant the clusters with bp > 95\% [37].

\section{Results and Discussion}

The sequencing resulted in a total of 19,433,337 sequences of the experimental group (Bap1-6,432,276; Bap2-8,113,328; and Bap3-4,887,733) and 20,802,491 sequences of the control group (Neg1-6,756,396; Neg2-5,724,941; and Neg3-8,321,154). Both groups were grown in the presence of glucose, whereas benzo(a)pyrene was only present in the experimental group. Even though several works choose to use the compound of interest as their sole source of carbon, we choose to use glucose as an additional carbon source for both optimizing growth and approximate environment conditions. Previous unpublished experiments from our group already demonstrate the ability of Burkholderia vietnamiensis G4 to grow using benzo(a)pyrene as the sole source of carbon. However, the intended application of this bacterial ability is to use it for bioremediation, a condition in which we would hardly find a single carbon source.

Evaluating the generated sequences, as well as the expressed genes, cluster analysis by correlation demonstrated well-supported clusters (bootstrap probability $>90$ ), forming clades in both the experimental and control groups (Figure 1).

The sequences were mapped in the reference indexer built from the complete genome and its reference file for the genomic regions, finding 7,840 genes from the total described for the bacterium of 7,861 representing $99 \%$ coverage in the mapping of the generated reads. Of the 7,840 genes mapped, we found significant values of differential expression for 156 regions when comparing the experimental group to the control group (Supplementary Table 1). In Volcano (Figure 2), the significant genes for differential gene expression had a higher Fragments Per Kilobase Million (FPKM) value in the group of bacteria exposed to benzo(a) pyrene, totaling 88 higher expressed genes (Table 1), indicating greater expression of these genes in the experimental group. Of the genes expressed, we found 34 hypothetical proteins, and there were 13 genes expressed in clusters-for evaluation, they were considered as a single route of degradation.

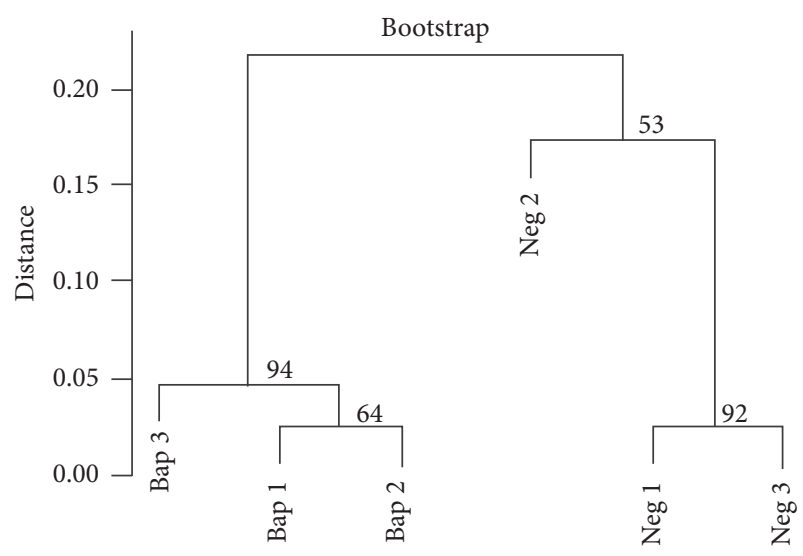

Figure 1: Gene-based correlation dendrogram between the experimental (Bap) and the control groups (Neg). The number in the branches indicates bootstrap support.

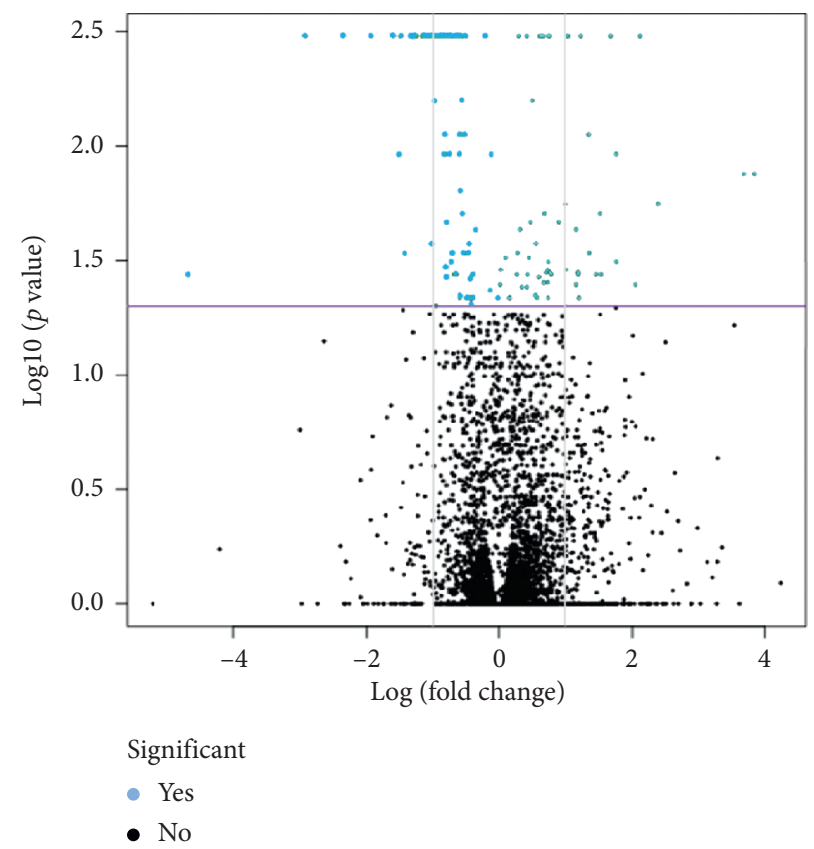

Figure 2: Volcano plot highlighting the genes differentially expressed between the group exposed to benzo(a)pyrene and the control group. A total of 156 differentially expressed genes $(p<0.05)$ were detected. In blue, we show the genes that presented statistically significant difference comparing the expression between both groups.

The differentially expressed genes observed in this study in the B. vietnamiensis strain G4 showed involvement in several functions of this microorganism, which is an indicative of high metabolic activity of this bacterium when in contact with benzo(a)pyrene. From the genetic functionalities found in the differently expressed genomic regions, several functional groups were found (hydrolases, oxidoreductase, lyases, and transferases). This diversity of expressed regions demonstrates high metabolic activity in the group exposed to benzo(a)pyrene. Among the candidate genes found, it was observed that they correspond to a variety of functional groups. Nevertheless, groups of genes 
characterized by their involvement in catalytic activities represented most differentially expressed genes (Figure 3). These enzymes are associated with diverse biochemical and metabolic pathways, as an integral part of some of the most important life processes of these organisms [39]. The success of degradation of PAHs by these enzymes is directly linked to the ionization potential of each molecule, which represents the energy required for the enzymes to be able to act by removing electrons from the aromatic rings, consequently reducing its structural stability leading to the formation of simpler compounds and/or the breakdown of these PAHs [40-42].

Eighty-eight genes were found to have significant differential expression in the experimental group. They were associated with more than one cellular route for the degradation of PAHs. We also found regulatory regions that do not allow the byproducts resulting from this breakdown, such as $\mathrm{CO}_{2}, \mathrm{H}_{2} \mathrm{O}$, ammonia, nitrogen, and potential charges of free electrons, which alter the intracellular $\mathrm{pH}$ and may lead to toxicity, to accumulate [43-45]. These regulatory regions provide the biological conversion of the toxic substrate generated by $\mathrm{PAHs}$ into some intermediate metabolites, such as acetyl-CoA, succinyl-CoA, and pyruvate, eliminating their cellular toxicity while maintaining their physiological functioning [46].

In order to reach successful degradation of PAHs, several cellular mechanisms must be active; thus, the expression of many functional groups, several proteins and enzymes, is expected. Among these, there are some membrane proteins that regulate the entry and exit of solutes and that were significantly highly expressed. Amid them, the major facilitator transporter (Bcep1808_2766) belongs to one of the largest super families of membrane transport proteins, also present in groups of bacteria, archaea, and eukaryotes. This family is important as an entry channel for micro- and macromolecules in the intracellular environment, as well as the release of dispensable substances [47], and may be active in the transport of benzo(a)pyrene molecules from the extracellular medium to the intracellular medium. The proteins amino acid ABC transporter (Bcep1808_0095, Bcep1808_3624, Bcep1808_5570, and Bcep1808_5571), oligopeptide/dipeptide ABC transport ATPase (Bcep1808_3703, Bcep1808_3703), glycine betaine/L-proline ABC transport ATPase (Bcep1808_3472), and ABC transporter (Bcep1808_5572) were all found to have a similar function. These proteins carry solutes and toxins through the membrane, regardless of the concentration gradient. Such movement occurs via ATP hydrolysis and it is fundamental to maintain cellular homeostasis [48]. Shuona et al. [49] found a positive regulation between transmembrane transport and the degradation of benzo(a)pyrene with the expression of certain proteins such as $\mathrm{ABC}$ transporter. The authors also highlight the relationship between the high hydrophobicity of the cell surface and the Gram-negative bacteria, such as B. vietnamiensis $\mathrm{G} 4$, which facilitates contact with benzo(a)pyrene.

Membrane activity is important for understanding the functioning and use of bacterial compounds. In this regard, a greater expression of the protein lysine exporter protein LysE/

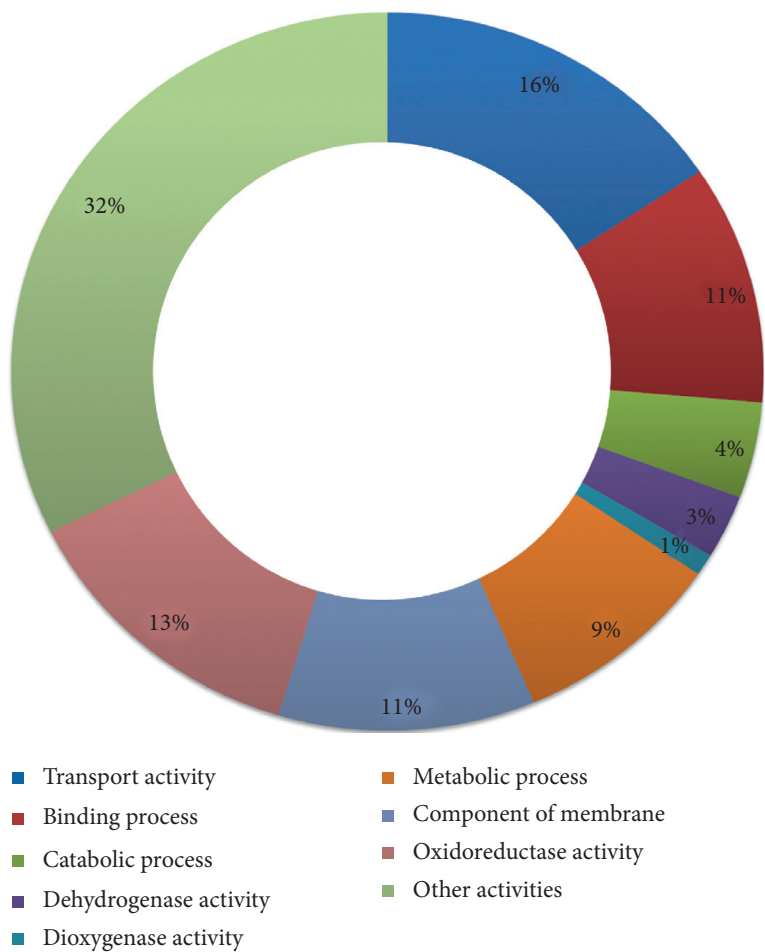

FIGURE 3: Distribution of genes differentially expressed by functional groups (GO Term).

YggA (Bcep1808_3754) was observed among the bacterial strains of the group exposed to benzo(a)pyrene. This family of proteins, according to Tsu and Saier [50], presents the capacity of transmembrane transportation of specific metals, such as nickel, iron, and magnesium. The authors also highlight the importance of cell homeostasis, regulating and protecting the cell from the accumulation of heavy metals, which may be an important factor for bacteria that live in environments affected by persistent organic and inorganic compounds.

In the groups of transport and membrane proteins with differential expression, it is worth noting the binding-protein-dependent transport system (Bcep1808_3704, Bcep1808_3705, and Bcep1808_3471), described by Higgins et al. [51], the extracellular solute-binding protein (Bcep1808_0449, Bcep1808_4396, Bcep1808_3706, and Bcep1808_5569), and the oxidoreductase FAD-binding subunit (Bcep1808_3415). The latter plays an important role in electron chain flow regulation, linked to a larger metabolic system where the use of enzymes such as dioxygenases stands out $[52,53]$. These enzymes form a super family of catalytic activity, and they are linked to the breakdown and transformation of organic compounds; the "Heterocyclic Ring Cleavage" [54] shows the importance of dioxygenases for the cleavage of aromatic rings and formation of carboncarbon bonds, which are essential for the degradation of benzo(a)pyrene. Wang et al. [55] also show the importance of dioxygenases in breaking the aromatic rings, as in their experiment, these proteins were highly expressed only in the group exposed to pyrene. Many bacterial strains use these enzymes to obtain energy and carbon for their biological 
processes [56]. They were first isolated and characterized in Pseudomonas putida [57], a phylogenetically species closely related species to $B$. vietnamiensis, and the increased expression of this oxidoreductase is another indication of the use of benzo(a)pyrene as an energy source by B. vietnamiensis. Within the group of oxidoreductases, increased gene expression was detected for the proteins glutamate synthase (Bcep1808_0384, Bcep1808_0385), methylitaconate delta2-delta3-isomerase (Bcep1808_3677), aldo/keto reductase (Bcep1808_3756), and FAD-dependent oxidoreductase (Bcep1808_0754).

Regions also linked to dioxygenases, such as 4-hydroxyphenylpyruvate-dioxygenase (Bcep1808_0303) and indolepyruvate-ferredoxin-oxidoreductase (Bcep1808_0301), were already described in studies of bacteria surviving in stressful and contaminated environments [58, 59], expecting greater expression in the experimental group exposed to benzo(a) pyrene.

Among the overexpressed genes observed in the presence of benzo(a)pyrene, gene products involved in important catalytic pathways of $\mathrm{PAH}$ degradation were obtained; some were already found in the study of Ma et al. [60]. These genes belong to the dehydrogenase groups: Acyl-CoA dehydrogenase (Bcep1808_3337), aldehyde dehydrogenase (Bcep1808_6786), methylmalonate-semialdehyde dehydrogenase (Bcep1808_3335), and trifunctional transcriptional regulator/proline dehydrogenase/pyrroline-5-carboxylate dehydrogenase (Bcep1808_0122). Proline is directly related to the presence of a stressing agent, in the case of benzo(a) pyrene, and its high expression contributes to the resistance of the microorganism in the medium [61]. In a study conducted by O'Sullivan et al. [18] with mutants for phenol degradation, genes such as aldehyde dehydrogenase were interrupted, which showed an effect on phenol degradation. Changes in these enzymes indicated that they have an important support for this function. The fact that this route is also more expressed in the groups in contact with benzo(a) pyrene reinforces the hypothesis of using these enzymes as necessary for the degradation of organic aromatic compounds in these bacterial groups [62,63].

The phenylacetic acid degradation protein is at the center of a route in the catabolism of aromatic compounds, converting the breakdown of these rings into energy directly in the Krebs cycle with the help of enzymes such as phenylacetate-CoA ligase [64-66]. In this study, we found four differentially expressed genes linked to this region: phenylacetate-CoA ligase (Bcep1808_0516), phenylacetate-CoA oxygenase/reductase subunit PaaK (Bcep1808_0308), phenylacetic acid degradation protein PaaD (Bcep1808_0517), and phenylacetic acid degradation protein paaN (Bcep1808_052). Therefore, the high expression of that route also places it as a strong candidate for benzo(a)pyrene degradation capacity [67] of B. vietnamiensis to obtain energy.

An important genomic region responsible for signaling degradation is the XRE family transcriptional regulator (xenobiotic response element) (Bcep1808_5376). This element is frequent in the regulation and activation of many metabolic pathways in bacteria and is involved in fitness and survival to stressful environments [68-70]. It is also linked to the activation of several pathways with capacity to use toxic compounds [71] active in bacteria when in contact with these compounds, such as the one used in the experiment, activating a system called toxin-antitoxin, which has a cascading effect that can generate production of biofilm, membrane enzymes, proteins for protection, growth, and cell signaling [72]. Considering that this is an element of resistance and activation of metabolic pathways, its increased expression makes it a good candidate to regulate the degradation of PAHs.

Four hypothetical proteins found are considered by us to represent the new candidates detected to play an important role in the degradation of benzo(a)pyrene. Searching for analogies and super families, the functional domain Dodecin was found for one of the hypothetical proteins (Bcep1808_1880). This domain is linked to small membrane molecules of the flavoproteins group, active in oxireduction and disposal pathways of cellular material $[73,74]$. They are linked to processes of oxireduction of pyruvate, fatty acids and in the electron transport in energy chains. The biotransformation of PAHs involves a series of enzymes that catalyze oxidation, reduction, and hydrolysis reactions and enzymes that catalyze conjugation reactions. Xu et al. [75] indicate that dioxygenase can be used as biomarkers to assess initial oxidation and attenuation of HPAs and in their work naphthalene dioxygenase is used for this purpose. In general, the degradation of benzo(a)pyrene produces active intermediate metabolites such as dihydrodiol and dihydrodiolepoxide $[76,77]$. Such transformation can be interpreted as a facilitator of excretions by making a given metabolite more hydrophilic than its precursor. The induction of metabolic pathways such as these by chemicals, in the specific case by benzo(a)pyrene, may be suggestive of the participation of this hypothetical protein in the degradation of this $\mathrm{PAH}$.

In studies involving benzo(a)pyrene degradation $[1,78-87]$, a great diversity of pathways, proteins and enzymes, are described. Among these, two large groups, dioxygenases and dihydrodiol, are the most cited as important in the degradation of benzo(a)pyrene as well as pyrene in the study of Wang et al. [55]. In our study, we found indications for the expression of dioxygenases. Meanwhile, the dihydrodiol groups did not show greater expression in this experiment, and it is speculated that this may be because these molecules are also expressed in the control group, even though it is not in contact with benzo(a) pyrene. We considered their involvement in other metabolic pathways and/or they might need longer than 45 minutes of exposure to be signaled and produced as possibilities for the nonsignificant expression of the dihydrodiol group in B. vietnamiensis $\mathrm{G} 4$.

\section{Conclusion}

In this study, the B. vietnamiensis $\mathrm{G} 4$ bacterium was exposed to the PAH benzo(a)pyrene, and the genes differentially expressed when in contact with this compound were evaluated. A total of 156 differentially expressed genes were found, 88 of which showed higher expression in the 
TABLE 1: Genes significantly expressed in the experimental group exposed to benzo(a)pyrene.

\begin{tabular}{|c|c|c|c|c|c|c|}
\hline $\begin{array}{l}\text { UniProtKB } \\
\text { accession }\end{array}$ & $\begin{array}{c}\text { NCBI old locus } \\
\text { Tag }\end{array}$ & Coordinates & $\begin{array}{l}\text { Gene } \\
\text { length }\end{array}$ & $\begin{array}{l}\text { Gene } \\
\text { name }\end{array}$ & Product description & $\begin{array}{c}\text { Significant in } \\
\text { BAP }\end{array}$ \\
\hline A4JK62 & Bcep1808_3680 & $427848 . .428741(--)$ & 894 & prpB & 2-Methylisocitrate lyase & Yes \\
\hline A4JAP4 & Bcep1808_0334 & $378768 . .379043(+)$ & 276 & rpsS & 30S ribosomal protein S19 & Yes \\
\hline A4JJM7 & Bcep1808_3492 & $203642 . .205642(-)$ & 2001 & & $\begin{array}{l}\text { 3-Methylcrotonoyl-CoA carboxylase } \\
\text { subunit alpha }\end{array}$ & Yes \\
\hline A4JDT5 & Bcep1808_1430 & $1537771 . .1538475(+)$ & 705 & & 3-Oxoacid CoA-transferase subunit A & Yes \\
\hline A4JAL3 & Bcep1808_0303 & $336630 . .337727(-)$ & 1098 & hppD & 4-Hydroxyphenylpyruvate dioxygenase & Yes \\
\hline A4JBB7 & Bcep1808_0558 & $614619 . .614882(+)$ & 264 & rpmA & 50 S ribosomal protein L27 & Yes \\
\hline A4JQF7 & Bcep1808_5572 & $122728 . .123519(+)$ & 792 & & $\mathrm{ABC}$ transporter related & Yes \\
\hline A4JA13 & Bcep1808_0093 & $100965 . .101744(+)$ & 780 & & $\mathrm{ABC}$ transporter-like protein & Yes \\
\hline A4JA20 & Bcep1808_0100 & $109204 . .110988(+)$ & 1785 & & $\mathrm{ABC}$ transporter-like protein & Yes \\
\hline A4JQ98 & Bcep1808_5507 & 63088..64278(+) & 1191 & & Acetyl-CoA acetyltransferase & Yes \\
\hline A4JK60 & Bcep1808_3678 & $423951 . .426545(--)$ & 2595 & & Aconitate hydratase & Yes \\
\hline A4JJ73 & Bcep1808_3337 & $29534 . .30667(-)$ & 1134 & & Acyl-CoA dehydrogenase & Yes \\
\hline A4JTS0 & Bcep1808_6786 & $182448 . .183836(-)$ & 1389 & & Aldehyde dehydrogenase & Yes \\
\hline A4JKD6 & Bcep1808_3756 & $501291 . .502235(+)$ & 945 & & aldo/keto reductase & Yes \\
\hline A4JJ72 & Bcep1808_3336 & $27598 . .29310(-)$ & 1713 & & AMP-dependent synthetase/ligase & Yes \\
\hline A4JQ96 & Bcep1808_5505 & 60596..62263(+) & 1668 & & AMP-dependent synthetase/ligase & Yes \\
\hline A4JCX9 & Bcep1808_1121 & $1218744 . .1219214(+)$ & 471 & & AsnC family transcriptional regulator & Yes \\
\hline A4JB78 & Bcep1808_0519 & $561462 . .562664(-)$ & 1203 & & Beta-ketoadipyl CoA thiolase & Yes \\
\hline A4JJK6 & Bcep1808_3471 & $173048 . .173944(-)$ & 897 & & $\begin{array}{l}\text { Binding-protein-dependent transport } \\
\text { system inner membrane protein }\end{array}$ & Yes \\
\hline A4JK86 & Bcep1808_3704 & $454654 . .455556(-)$ & 903 & & $\begin{array}{l}\text { Binding-protein-dependent transport } \\
\text { system inner membrane protein }\end{array}$ & Yes \\
\hline A4JK87 & Bcep1808_3705 & $455570 . .456508(-)$ & 939 & & $\begin{array}{l}\text { Binding-protein-dependent transport } \\
\text { system inner membrane protein }\end{array}$ & Yes \\
\hline
\end{tabular}

experimental group. Such high number of genes expressed suggests the possibility of several pathways of degradation of this compound. Considering that PAHs are persistent and highly harmful pollutants to human health and the environment, benzo(a)pyrene as one of the most toxic, the optimization of bioremediation processes is of fundamental importance $[1,2]$. The mitigation of effects of these compounds in nature is essential for the conservation of ecosystems that are increasingly affected by PAHs, due to a growing use of oil derivatives and consequent increase of the global demand for their refining and processing, generating higher quantities of these compounds disposed in nature. Experiments with exposure of bacteria with capacity for degradation of persistent compounds, such as benzo(a) pyrene, test bacterial strains against model residual elements, thus verifying the ability of these compounds to be used by these microorganisms. The differential expression tests are informative to understand this bacterial capacity to degrade/assimilate and use these compounds in their metabolic pathways.

The evidence found in this study reveals some candidate genes for the degradation capacity of benzo(a)pyrene, a compound considered of difficult degradation. Understanding these processes can be useful for future efforts of increased degradation through genetic engineering and controlled overexpression of target genes.

\section{Data Availability}

The underlying data supporting the results of their study, in addition to the supplementary materials available in the submitted document, are available and can be requested by sending e-mails to valiati@unisinos.br and gcauduro@ gmail.com.

\section{Conflicts of Interest}

The authors declare that there are no conflicts of interest regarding the publication of this paper.

\section{Acknowledgments}

The authors gratefully acknowledge the Coordenação de Aperfeiçoamento de Pessoal de Nível Superior (CAPES, Brazil) for research grants and fellowships in support of this study, Conselho Nacional de Desenvolvimento Científico e Tecnológico (CNPq) for research grants to VHV (308996/ 2017-8), and Programa de Pós-Graduação em Biologia (UNISINOS). The authors thank the Companhia Riograndense de Saneamento (CORSAN), for providing samples and laboratory equipment for the realization of this study. Credit goes to the operators of the Laboratory of Genetics and Molecular Biology (LGBM/UNISINOS) Gabriela Kern and Renan Cesar Sbruzzi and for the technicians. The work had financial support only for the acquisition of some laboratory equipment that helped to carry out this study. In addition, the Coordenação de Aperfeiçoamento de Pessoal de Nível Superior (CAPES, Brazil) provided the scholarship for a Ph.D. student. All other inputs for the different techniques were funded by the project coordinator's own funds. 


\section{Supplementary Materials}

Supplementary Table 1 . The 156 genes that were found with significant values of differential expression when we compared the experimental group with the control group. (Supplementary Materials)

\section{References}

[1] D. Ghosal, S. Ghosh, T. K. Dutta, and Y. Ahn, "Current state of knowledge in microbial degradation of polycyclic aromatic hydrocarbons (PAHs): a review," Frontiers in Microbiology, vol. 7, p. 1369, 2016.

[2] R. M. C. W. Tonini, C. E. Rezende, and A. D. Grativol, "Degradação e biorremediação de compostos do petróleo por bactérias: revisão," Oecologia Australis, vol. 14, no. 4, pp. 1025-1035, 2010.

[3] D. Mackay and D. Callcott, "Partitioning and physical chemical properties of PAHs," The Handbook of Environmental Chemistry, pp. 325-345, 1998.

[4] A. L. Juhasz and R. Naidu, "Bioremediation of high molecular weight polycyclic aromatic hydrocarbons: a review of the microbial degradation of benzo[a]pyrene," International Biodeterioration \& Biodegradation, vol. 45, no. 1-2, pp. 57-88, 2000.

[5] C. P. Marston, C. Pereira, J. Ferguson et al., "Effect of a complex environmental mixture from coal tar containing polycyclic aromatic hydrocarbons $(\mathrm{PAH})$ on the tumor initiation, PAH-DNA binding and metabolic activation of carcinogenic PAH in mouse epidermis," Carcinogenesis, vol. 22, no. 7, pp. 1077-1086, 2001.

[6] K. Watanabe, H. Futamata, and S. Harayama, Antonie Van Leeuwenhoek, vol. 81, no. 1/4, pp. 655-663, 2002.

[7] Z. Cui, Q. Lai, C. Dong, and Z. Shao, "Biodiversity of polycyclic aromatic hydrocarbon-degrading bacteria from deep sea sediments of the Middle Atlantic Ridge," Environmental Microbiology, vol. 10, no. 8, pp. 2138-2149, 2008.

[8] W. Qin, F. Fan, Y. Zhu et al., "Comparative proteomic analysis and characterization of benzo(a)pyrene removal by Microbacterium sp. strain M.CSW3 under denitrifying conditions," Bioprocess and Biosystems Engineering, vol. 40, no. 12, pp. 1825-1838, 2017.

[9] A. K. Haritash, "A comprehensive review of metabolic and genomic aspects of PAH-degradation," Archives of Microbiology, 2020.

[10] Y. Ma, L. Wang, and Z. Shao, "Pseudomonas, the dominant polycyclic aromatic hydrocarbon-degrading bacteria isolated from Antarctic soils and the role of large plasmids in horizontal gene transfer," Environmental Microbiology, vol. 8, no. 3, pp. 455-465, 2006.

[11] C. Liang, Y. Huang, and H. Wang, "pahE, a functional marker gene for polycyclic aromatic hydrocarbon-degrading bacteria," Applied and Environmental Microbiology, vol. 85, no. 3, 2018.

[12] S. Fields, "CELL BIOLOGY: whither model organism research?” Science, vol. 307, no. 5717, pp. 1885-1886, 2005.

[13] V. De Gannes and W. J. Hickey, "Genetic adaptations of bacteria for metabolism of polycyclic aromatic hydrocarbons," in Microbial Ecotoxicology, pp. 133-164, Springer, Cham, Switzerland, 2017.

[14] X.-x. Liu, X. Hu, Y. Cao et al., "Biodegradation of phenanthrene and heavy metal removal by acid-tolerant Burkholderia fungorum FM-2," Frontiers in Microbiology, vol. 10, 2019.
[15] J. Caballero-Mellado, J. Onofre-Lemus, P. Estrada-de los Santos, and L. Martinez-Aguilar, "The tomato rhizosphere, an environment rich in nitrogen-fixing Burkholderia species with capabilities of interest for agriculture and bioremediation," Applied and Environmental Microbiology, vol. 73, no. 16, pp. 5308-5319, 2007.

[16] X. Xiao, H. Chen, C. Si, and L. Wu, "Influence of biosurfactant-producing strain Bacillus subtilis BS1 on the mycoremediation of soils contaminated with phenanthrene," International Biodeterioration \& Biodegradation, vol. 75, pp. 36-42, 2012.

[17] W. Liu, J. Sun, L. Ding, Y. Luo, M. Chen, and C. Tang, "Rhizobacteria (Pseudomonas sp. SB) assist phytoremediation of oily-sludge-contaminated soil by tall fescue (Testuca arundinacea L.)," Plant and Soil, vol. 371, no. 1-2, pp. 533-542, 2013.

[18] L. A. O’Sullivan, A. J. Weightman, T. H. Jones, A. M. Marchbank, J. M. Tiedje, and E. Mahenthiralingam, "Identifying the genetic basis of ecologically and biotechnologically useful functions of the bacterium Burkholderia vietnamiensis," Environmental Microbiology, vol. 9, no. 4, pp. 1017-1034, 2007.

[19] E. Mahenthiralingam, T. A. Urban, and J. B. Goldberg, "The multifarious, multireplicon Burkholderia cepacia complex," Nature Reviews Microbiology, vol. 3, no. 2, pp. 144-156, 2005.

[20] M. J. Nelson, S. O. Montgomery, W. R. Mahaffey, and P. H. Pritchard, "Biodegradation of trichloroethylene and involvement of an aromatic biodegradative pathway," Applied and Environmental Microbiology, vol. 53, no. 5, pp. 949-954, 1987.

[21] R. Morya, D. Salvachúa, and I. S. Thakur, "Burkholderia: an untapped but promising bacterial genus for the conversion of aromatic compounds," Trends in Biotechnology, 2020.

[22] A. Nzila, S. Sankaran, M. Al-Momani, and M. M. Musa, "Isolation and characterization of bacteria degrading polycyclic aromatic hydrocarbons: phenanthrene and anthracene," Archives of Environmental Protection, vol. 44, 2018.

[23] L. A. O’Sullivan and E. Mahenthiralingam, "Biotechnological potential within the genus Burkholderia," Letters in Applied Microbiology, vol. 41, no. 1, pp. 8-11, 2005.

[24] G. L. Winsor, B. Khaira, T. Van Rossum, R. Lo, M. D. Whiteside, and F. S. L. Brinkman, "The Burkholderia Genome Database: facilitating flexible queries and comparative analyses," Bioinformatics, vol. 24, no. 23, pp. 2803-2804, 2008.

[25] R. Weiser, Z. L. Yap, A. Otter et al., "A novel inducible prophage from Burkholderia vietnamiensis G4 is widely distributed across the species and has lytic activity against pathogenic Burkholderia," Viruses, vol. 12, no. 6, p. 601, 2020.

[26] K. Wei, H. Yin, H. Peng, Z. Liu, G. Lu, and Z. Dang, "Characteristics and proteomic analysis of pyrene degradation by Brevibacillus brevis in liquid medium," Chemosphere, vol. 178, pp. 80-87, 2017.

[27] S. Yan and G. Wu, "Reorganization of gene network for degradation of polycyclic aromatic hydrocarbons (PAHs) in Pseudomonas aeruginosa PAO1 under several conditions," Journal of Applied Genetics, vol. 58, no. 4, pp. 545-563, 2017.

[28] Q. Ma, Z. Liu, B. Yang, C. Dai, and Y. Qu, "Characterization and functional gene analysis of a newly isolated indoledegrading bacterium Burkholderia sp. IDO3," Journal of Hazardous Materials, vol. 367, pp. 144-151, 2019.

[29] E. F. Lopes, J. G. Da Costa, I. R. Wolf, J. P. d. A. Lima, and S. Astolfi-Filho, "Draft genome sequence of Burkholderia gladioli Coa14, a bacterium with petroleum bioremediation 
potential isolated from coari Lake, amazonas, Brazil," Genome Announcements, vol. 6, no. 16, pp. e00301-18, 2018.

[30] S. Vikram, J. Pandey, N. Bhalla et al., "Branching of the p-nitrophenol (PNP) degradation pathway in Burkholderia sp. Strain SJ98: evidences from genetic characterization of PNP gene cluster," AMB Express, vol. 2, no. 1, p. 30, 2012.

[31] D. R. Yoder-Himes, P. S. G. Chain, Y. Zhu et al., "Mapping the Burkholderia cenocepacia niche response via high-throughput sequencing," Proceedings of the National Academy of Sciences, vol. 106, no. 10, pp. 3976-3981, 2009.

[32] R. Gao, C. Hao Da, W. Hu, S. Song, S. Y. Li, and G. B. Ge, "Transcriptome profile of polycyclic aromatic hydrocarbondegrading fungi isolated from Taxus rhizosphere," Current Science, vol. 116, no. 7, 2019.

[33] B. Ewing and P. Green, "Base-calling of automated sequencer traces UsingPhred.II. Error probabilities," Genome Research, vol. 8, no. 3, pp. 186-194, 1998.

[34] S. Andrews, "FastQC: a quality control tool for high throughput sequence data," September 2019, http://www. bioinformatics.babraham.ac.uk/projects/fastqc.

[35] C. Trapnell, B. A. Williams, G. Pertea et al., "Transcript assembly and quantification by RNA-Seq reveals unannotated transcripts and isoform switching during cell differentiation," Nature Biotechnology, vol. 28, no. 5, pp. 511-515, 2010.

[36] C. Trapnell, A. Roberts, L. Goff et al., "Differential gene and transcript expression analysis of RNA-seq experiments with TopHat and Cufflinks," Nature Protocols, vol. 7, no. 3, pp. 562-578, 2012.

[37] R. Suzuki and H. Shimodaira, "Pvclust: an R package for assessing the uncertainty in hierarchical clustering," Bioinformatics, vol. 22, no. 12, pp. 1540-1542, 2006.

[38] F. D. Gibbons, "Judging the quality of gene expression-based clustering methods using gene annotation," Genome Research, vol. 12, no. 10, pp. 1574-1581, 2002.

[39] F. H. Arnold, "Directed evolution: bringing new chemistry to life," Angewandte Chemie International Edition, vol. 57, no. 16, pp. 4143-4148, 2017.

[40] P. Giovanella, G. A. L. Vieira, I. V. Ramos Otero, E. Pellizzer, B. de Jesus Fontes, and L. D. Sette, "Metal and organic pollutants bioremediation by extremophile microorganisms," Journal of Hazardous Materials, p. 121024, 2019.

[41] B. Sharma, A. K. Dangi, and P. Shukla, "Contemporary enzyme based technologies for bioremediation: a review," Journal of Environmental Management, vol. 210, pp. 10-22, 2018.

[42] X.-X. Zhang, S.-P. Cheng, C.-J. Zhu, and S.-L. Sun, "Microbial $\mathrm{PAH}$-degradation in soil: degradation pathways and contributing factors," Pedosphere, vol. 16, no. 5, pp. 555-565, 2006.

[43] S. H. Light, L. Su, R. Rivera-Lugo et al., "A flavin-based extracellular electron transfer mechanism in diverse Grampositive bacteria," Nature, 2018.

[44] K. H. Hansen, I. Angelidaki, and B. K. Ahring, "Anaerobic digestion of swine manure: inhibition by ammonia," Water Research, vol. 32, no. 1, pp. 5-12, 1998.

[45] M. Vermuë, J. Sikkema, A. Verheul, R. Bakker, and J. Tramper, "Toxicity of homologous series of organic solvents for the gram-positive bacteriaArthrobacterandNocardiaSp. and the gram-negative bacteriaAcinetobacterandPseudomonasSp," Biotechnology and Bioengineering, vol. 42, no. 6, pp. 747-758, 1993.

[46] S. Y. Lee, S. S. Sekhon, Y.-H. Ban et al., "Proteomic analysis of polycyclic aromatic hydrocarbons (PAHs) degradation and detoxification in sphingobium chungbukense DJ77," Journal of Microbiology and Biotechnology, vol. 26, no. 11, pp. 19431950, 2016.

[47] S. S. Pao, I. T. Paulsen, and M. H. Saier Jr., "Major facilitator superfamily," Microbiology and Molecular Biology Reviews, vol. 62, no. 1, pp. 1-34, 1998.

[48] P. M. Jones and A. M. George, "The ABC transporter structure and mechanism: perspectives on recent research," Cellular and Molecular Life Sciences (CMLS), vol. 61, no. 6, pp. 682699, 2004.

[49] C. Shuona, Y. Hua, C. Jingjing, P. Hui, and D. Zhi, "Physiology and bioprocess of single cell of Stenotrophomonas maltophilia in bioremediation of co-existed benzo[a]pyrene and copper," Journal of Hazardous Materials, vol. 321, pp. 9-17, 2017.

[50] B. V. Tsu and M. H. Saier, "The LysE superfamily of transport proteins involved in cell physiology and pathogenesis," PLoS One, vol. 10, no. 10, 2015.

[51] C. F. Higgins, M. P. Gallagher, S. C. Hyde et al., "Periplasmic binding protein-dependent transport systems: the membraneassociated components [and discussion]," Philosophical Transactions of the Royal Society B: Biological Sciences, vol. 326, no. 1236, pp. 353-365, 1990.

[52] C. Liang, Y. Huang, Y. Wang, Q. Ye, Z. Zhang, and H. Wang, "Distribution of bacterial polycyclic aromatic hydrocarbon (PAH) ring-hydroxylating dioxygenases genes in oilfield soils and mangrove sediments explored by gene-targeted metagenomics," Applied Microbiology and Biotechnology, 2019.

[53] J. R. Mason and R. Cammack, "The electron-transport proteins of hydroxylating bacterial dioxygenases," Annual Review of Microbiology, vol. 46, no. 1, pp. 277-305, 1992.

[54] Y. Zhu, K. Chen, Y. Ding et al., "Metabolic and proteomic mechanism of benzo[a]pyrene degradation by Brevibacillus brevis," Ecotoxicology and Environmental Safety, vol. 172, pp. 1-10, 2019.

[55] W. Wang, L. Wang, and Z. Shao, "Polycyclic aromatic hydrocarbon (PAH) degradation pathways of the obligate marine PAH degrader Cycloclasticus sp. Strain P1," Appl Environ Microbiol, vol. 84, pp. e01261-18, 2018.

[56] M. Holmquist, "Alpha beta-hydrolase fold enzymes structures, functions and mechanisms," Current Protein and Peptide Science, vol. 1, no. 2, pp. 209-235, 2000.

[57] I. Bauer, N. Max, S. Fetzner, and F. Lingens, "2,4-Dioxygenases catalyzing N-Heterocyclic-Ring cleavage and formation of carbon monoxide. Purification and some properties of $1 \mathrm{H}$ 3-Hydroxy-4-Oxoquinaldine 2,4-dioxygenase from arthrobacter sp. Ru61a and comparison with 1H-3-Hydroxy-4Oxoquinoline 2,4-dioxygenase from Pseudomonas putida 33/ 1," European Journal of Biochemistry, vol. 240, no. 3, pp. 576-583, 1996.

[58] D. Singh, J. Kumar, and A. Kumar, "Isolation of pyomelanin from bacteria and evidences showing its synthesis by 4 hydroxyphenylpyruvate dioxygenase enzyme encoded by hppD gene," International Journal of Biological Macromolecules, vol. 119, pp. 864-873, 2018.

[59] K. Wu, T.-H. Lee, Y.-L. Chen et al., "Identification of metabolites involved in the aerobic degradation of estrogen A/Brings," Applied and Environmental Microbiology, 2018.

[60] L. Ma, F. Deng, C. Yang, C. Guo, and Z. Dang, "Bioremediation of PAH-contaminated farmland: field experiment," Environmental Science and Pollution Research, vol. 25, no. 1, pp. 64-72, 2016.

[61] B. H. Gregson, G. Metodieva, M. V. Metodiev, P. N. Golyshin, and B. A. McKew, "Protein expression in the obligate hydrocarbon-degrading psychrophile Oleispira antarctica RB-8 
during alkane degradation and cold tolerance," Environmental Microbiology, vol. 22, no. 5, pp. 1870-1883, 2020.

[62] Y.-C. Chang, Microbial Biodegradation of Xenobiotic Compounds, CRC Press, Boca Raton, FL, USA, 1st edition, 2019.

[63] R. J. Law, J. N. R. Hamlin, A. Sivro, S. J. McCorrister, G. A. Cardama, and S. T. Cardona, "A functional phenylacetic acid catabolic pathway is required for full pathogenicity of Burkholderia cenocepacia in the Caenorhabditis elegans host model," Journal of Bacteriology, vol. 190, no. 21, pp. 7209-7218, 2008.

[64] W. Liu, J. Pan, X. Feng et al., "Evidence of aromatic degradation predominantly via phenylacetic acid in Marine Thermoprofundals," Environmental Microbiology, 2019.

[65] J. N. Hamlin, R. A. Bloodworth, and S. T. Cardona, "Regulation of phenylacetic acid degradation genes of Burkholderia cenocepacia K56-2," BMC Microbiology, vol. 9, no. 1, p. 222, 2009.

[66] S. Dalvi, C. Nicholson, F. Najar et al., “Arhodomonas sp. strain seminole and its genetic potential to degrade Aromatic compounds under high-salinity conditions," Applied and Environmental Microbiology, vol. 80, no. 21, pp. 6664-6676, 2014.

[67] R. Teufel, V. Mascaraque, W. Ismail et al., "Bacterial phenylalanine and phenylacetate catabolic pathway revealed," Proceedings of the National Academy of Sciences, vol. 107, no. 32, pp. 14390-14395, 2010.

[68] Y. Hu, Q. Hu, R. Wei et al., "The XRE family transcriptional regulator SrtR in Streptococcus suis is involved in oxidant tolerance and virulence," Front. Cell. Infect. Microbiol.vol. 8, p. $452,2019$.

[69] R. I. Santamaría, L. Sevillano, J. Martín, O. Genilloud, I. González, and M. Díaz, “The XRE-DUF397 protein pair, Scr1 and Scr2, acts as a strong positive regulator of antibiotic production in streptomyces," Frontiers in Microbiology, vol. 9, 2018.

[70] R. Page and W. Peti, "Toxin-antitoxin systems in bacterial growth arrest and persistence," Nature Chemical Biology, vol. 12, no. 4, pp. 208-214, 2016.

[71] T. R. Blower, G. P. Salmond, and B. F. Luisi, "Balancing at survival's edge: the structure and adaptive benefits of prokaryotic toxin-antitoxin partners," Current Opinion in Structural Biology, vol. 21, no. 1, pp. 109-118, 2011.

[72] X. Wang and T. K. Wood, "Toxin-antitoxin systems influence biofilm and persister cell formation and the general stress response," Applied and Environmental Microbiology, vol. 77, no. 16 , pp. 5577-5583, 2011.

[73] A. Nzila, C. O. Ramirez, M. M. Musa, S. Sankara, C. Basheer, and Q. X. Li, "Pyrene biodegradation and proteomic analysis in Achromobacter xylosoxidans, PY4 strain," International Biodeterioration \& Biodegradation, vol. 130, pp. 40-47, 2018.

[74] M. Grininger, K. Zeth, and D. Oesterhelt, "Dodecins: a family of lumichrome binding proteins," Journal of Molecular Biology, vol. 357, no. 3, pp. 842-857, 2006.

[75] Y. Xu, T. Liu, X. Zhu, and G. Ji, "Quantitative analysis of genetic associations in the biodegradative pathway of PAHs in wetland sediments of the Bohai coast region," Chemosphere, 2018.

[76] E. M. Ostrem Loss and J.-H. Yu, "Bioremediation and microbial metabolism of benzo(a)pyrene," Molecular Microbiology, 2018.

[77] C. E. Cerniglia, Advances in Applied Microbiology, Academic Press, San Diego, CA, USA, 1984.

[78] M. A. Heitkamp, J. P. Freeman, D. W. Miller, and C. E. Cerniglia, "Pyrene degradation by a Mycobacterium sp.: identification of ring oxidation and ring fission products," Applied and Environmental Microbiology, vol. 54, no. 10, pp. 2556-2565, 1988.

[79] J.-S. Seo, Y.-S. Keum, and Q. Li, "Bacterial degradation of aromatic compounds," International Journal of Environmental Research and Public Health, vol. 6, no. 1, pp. 278-309, 2009.

[80] J. A. Rentz, P. J. J. Alvarez, and J. L. Schnoor, "Benzo[a]pyrene degradation by Sphingomonas yanoikuyae JAR02," Environmental Pollution, vol. 151, no. 3, pp. 669-677, 2008.

[81] J. D. Moody, J. P. Freeman, P. P. Fu, and C. E. Cerniglia, "Degradation of benzo[a]pyrene by Mycobacterium vanbaalenii PYR-1," Applied and Environmental Microbiology, vol. 70, no. 1, pp. 340-345, 2004.

[82] M. Lozada, J. P. Riva Mercadal, L. D. Guerrero, W. D. Di Marzio, M. A. Ferrero, and H. M. Dionisi, "Novel aromatic ring-hydroxylating dioxygenase genes from coastal marine sediments of Patagonia," BMC Microbiology, vol. 8, no. 1, p. 50,2008 .

[83] D. Gibson, V. Mahadevan, D. Jerina, H. Yogi, and H. Yeh, "Oxidation of the carcinogens benzo [a] pyrene and benzo [a] anthracene to dihydrodiols by a bacterium," Science, vol. 189, no. 4199, pp. 295-297, 1975.

[84] I. Kelley, J. P. Freeman, F. E. Evans, and C. E. Cerniglia, "Identification of metabolites from the degradation of fluoranthene by Mycobacterium sp. Strain PYR-1," Applied and Environmental Microbiology, vol. 59, no. 3, pp. 800-880, 1993.

[85] F. Abbasian, R. Lockington, M. Megharaj, and R. Naidu, "A review on the genetics of aliphatic and aromatic hydrocarbon degradation," Applied Biochemistry and Biotechnology, vol. 178, no. 2, pp. 224-250, 2015.

[86] B. Chen, J. Huang, K. Yuan et al., "Direct evidences on bacterial growth pattern regulating pyrene degradation pathway and genotypic dioxygenase expression," Marine Pollution Bulletin, vol. 105, no. 1, pp. 73-80, 2016.

[87] D. T. Gibson and R. E. Parales, "Aromatic hydrocarbon dioxygenases in environmental biotechnology," Current Opinion in Biotechnology, vol. 11, no. 3, pp. 236-243, 2000. 